\title{
Can infant lung function predict respiratory morbidity during the first year of life in preterm infants?
}

\author{
Elena Proietti ${ }^{1,2,7}$, Thomas Riedel ${ }^{3,7}$, Oliver Fuchs ${ }^{1,4}$, Isabelle Pramana², \\ Florian Singer ${ }^{1,5}$, Anne Schmidt ${ }^{1,2}$, Claudia Kuehni ${ }^{6}$, Philipp Latzin ${ }^{1,2}$ and Urs Frey ${ }^{2}$
}

Affiliations: 'Paediatric Pulmonology, Inselspital and University of Bern, Bern, Switzerland. ${ }^{2}$ University Children's Hospital (UKBB), Basel, Switzerland. ${ }^{3}$ Paediatric Intensive Care, Inselspital and University of Bern, Switzerland. 'University Children's Hospital, Munich, Germany. ${ }^{5}$ University Children's Hospital, Zurich, Switzerland. ${ }^{6}$ Institute for Social and Preventive Medicine (ISPM), University of Bern, Bern, Switzerland. ${ }^{7}$ These authors contributed equally to this work.

Correspondence: Urs Frey, UKBB, Spitalstrasse 33, 4056 Basel, Switzerland. E-mail: urs.freyवukbb.ch

ABSTRACT Compared with term-born infants, preterm infants have increased respiratory morbidity in the first year of life. We investigated whether lung function tests performed near term predict subsequent respiratory morbidity during the first year of life and compared this to standard clinical parameters in preterms.

The prospective birth cohort included randomly selected preterm infants with and without bronchopulmonary dysplasia. Lung function (tidal breathing and multiple-breath washout) was measured at 44 weeks post-menstrual age during natural sleep. We assessed respiratory morbidity (wheeze, hospitalisation, inhalation and home oxygen therapy) after 1 year using a standardised questionnaire. We first assessed the association between lung function and subsequent respiratory morbidity. Secondly, we compared the predictive power of standard clinical predictors with and without lung function data.

In 166 preterm infants, tidal volume, time to peak tidal expiratory flow/expiratory time ratio and respiratory rate were significantly associated with subsequent wheeze. In comparison with standard clinical predictors, lung function did not improve the prediction of later respiratory morbidity in an individual child.

Although associated with later wheeze, noninvasive infant lung function shows large physiological variability and does not add to clinically relevant risk prediction for subsequent respiratory morbidity in an individual preterm.

@ERSpublications

Infant lung function tests do not help to predict symptoms in preterms but show early pathophysiological alterations http://ow.ly/uyJpW

This article has supplementary material available from www.erj.ersjournals.com

Received: Aug 272013 | Accepted after revision: March 072014 | First published online: April 032014

Support statement: This work was supported by the Swiss National Science Foundation (grants 1209932473B_124654 and 324730_144280 to E. Proietti, O. Fuchs, A. Schmidt, P. Latzin and U. Frey). The funder had no role in study design, data collection and analysis, decision to publish, or preparation of the manuscript.

Conflict of interest: None declared.

Copyright @ERS 2014 


\section{Introduction}

Respiratory morbidity is a common consequence of prematurity. Compared with term-born infants, preterm infants are more likely to have lung function alterations, and to develop respiratory symptoms in early infancy, childhood and adulthood [1-5]. Several studies identified various perinatal factors leading to impaired lung function (e.g. post menstrual age (PMA) at birth or days on supplemental oxygen) and/or respiratory symptoms in later life (e.g. perinatal adaptation or ventilator support) [2,5-7]. Although infants with bronchopulmonary dysplasia (BPD) [8] are at higher risk of pulmonary morbidity, neither standard perinatal clinical risk predictors nor BPD severity classification can reliably predict subsequent respiratory disease burden in the first year of life for an individual child $[7,9]$.

For clinical management, we need early functional biomarkers to identify infants with particular risks of subsequent respiratory morbidity. Infant lung function has been proposed as such a biomarker predicting respiratory morbidity in early life [10]. So far, few studies have investigated the association between early lung function and clinical outcome in the first years of life. Most of these studies included babies born at or near term [11-15]. For use in daily clinical routine, lung function measurements should not require sedation, and be minimally invasive and easily applicable at bedside, and normative values should be available. We developed such a lung function test compatible with the latest European Respiratory Society (ERS)/American Thoracic Society (ATS) consensus statement in our cohort of healthy infants [16-18].

The aims of the present study were to investigate in infants born prematurely: first, to determine whether noninvasive lung function measured near term (44 weeks PMA) is associated with subsequent respiratory morbidity during the first year of life (pathophysiological association); and second, to assess the usefulness of early lung function tests to predict later respiratory morbidity in the individual (clinical usefulness). In particular, we investigated whether postnatal lung function adds to the predictive power for subsequent symptoms in addition to standard perinatal predictors and risk scores.

\section{Methods}

Study design

We prospectively recruited preterm infants during postnatal hospitalisation and performed lung function measurements at the age of 44 weeks PMA [2]. After 1 year of age, we sent standardised questionnaires to the parents to record respiratory symptoms during the first year of life [7]. The Ethics Committee of the Canton of Bern (Bern, Switzerland) approved the study protocol and parents agreed, with written consent, to participate in the study.

\section{Subjects}

Infants born prematurely ( $<37$ weeks PMA) were recruited at the University Children's Hospital of Bern (Bern, Switzerland) between 1999 and 2010, excluding infants with congenital diaphragmatic hernia. Data on perinatal course were recorded using the neonatology database (NEODAT 4.10, Tubingen, Germany). We additionally assessed commonly used clinical scores such as the clinical risk index for babies (CRIB) [19] and the ATS Jobe-Bancalari BPD severity classification [8].

\section{Lung function measurement}

At the age of 44 weeks PMA, we performed lung function tests in unsedated infants during non-rapid eye movement sleep (by monitoring behaviour and online flow-volume curves). First, we measured tidal breathing for $10 \mathrm{~min}$, assessing tidal volume $(V \mathrm{~T})$, respiratory rate, minute ventilation $\left(V^{\prime} \mathrm{E}\right)$, tidal expiratory flows at 25\% (TEF25) and 50\% (TEF50) of the remaining $V \mathrm{~T}$, peak flow, and the time to peak tidal expiratory flow ( $t \mathrm{PTEF}) /$ expiratory time $(t \mathrm{E})$ ratio, the latter describing the shape of the tidal breathing flow-volume loop. Second, we applied triplicate multiple-breath washout (MBW) measurements (each lasting 3-4 min) using 4\% sulfur hexafluoride to estimate functional residual capacity (FRC) and ventilation heterogeneity, assessing lung clearance index (LCI), and first and second moment ratios. LCI is defined as the number of lung volume turnovers (in terms of FRC) required to reduce the sulfur hexafluoride to $1 / 40$ th of the starting concentration. It is calculated as cumulative expired volume/FRC. During the measurements, we aimed for a transcutaneous oxygen saturation $>92 \%$. When infants required supplemental oxygen, we started the analysis from the first constant breaths. Measurements were not undertaken in children whose oxygen saturation fell below $92 \%$ while breathing room air. We performed the measurements and the analysis according to the ERS/ATS consensus, using an ultrasonic flow meter device (Exhalyzer D; Eco Medics AG, Duernten, Switzerland), as previously reported [16]. The equipment's dynamic dead space is estimated to be far below the recommended $2 \mathrm{~mL} \cdot \mathrm{kg}^{-1}$. 


\section{Outcomes and questionnaire}

This preterm cohort (recruitment period 1999-2007) has been described in detail in two previous papers [2,7]. Since then, recruitment has been ongoing. The present study included infants recruited from January 1999 to December 31 2010. Follow-up questionnaires for the additional participants were distributed in 2011.

The questionnaire contained questions modified from that of the International Study on Asthma and Allergies in Childhood (http://isaac.auckland.ac.nz) (online supplement material). We defined four binary outcomes (yes/no): wheeze, inhalation therapy with $\beta$-agonists for $>4$ weeks, re-hospitalisation due to respiratory disease (excluding admissions for diagnostic purposes), and home oxygen therapy after hospital discharge. These outcomes can be reproducibly assessed, as previously reported in a subpopulation of this cohort and in other studies [7, 20,21]. The returned questionnaires underwent quality control performed by study nurses and medical doctors. We further validated the answers for re-hospitalisation and home oxygen therapy using medical histories from hospital records. As four out of 45 re-hospitalisations were related to check-ups rather than symptoms, these were recoded.

\section{Statistical analysis}

Pathophysiological association

We investigated the association between lung function parameters and subsequent respiratory morbidity separately for each of the four outcomes, using multivariable logistic regression. Adverse perinatal course and pre-existing risk factors may cause respiratory morbidity through an independent pathway not involving lung function. Therefore, to avoid confounding in the association between lung function and respiratory symptoms, we adjusted the models for: maternal atopy (defined as history of hay fever, asthma or eczema); sex; mode of delivery; PMA at birth; Apgar score at $5 \mathrm{~min}$; duration of mechanical ventilation (days of invasive ventilation (intubation) plus days of noninvasive ventilation (continuous positive airway pressure)); weight gain as a standard deviation score (SDS) using the 1990 British Growth Reference from birth until the lung function test date; and PMA, weight in SDS and length in centimetres at the lung function test date. To reduce the risk of collinearity and Type I error, we chose the previously mentioned clinical parameters prior to statistical analysis, guided by clinical evidence [9] and experience from our prospective healthy term cohort from the same region $[7,22]$. We describe sensitivity analyses in the online supplementary material.

\section{Clinical relevance}

We investigated whether the performed lung function test improved the prediction of respiratory symptoms compared to the standard clinical predictors or scores alone. We carried out logistic regressions using common clinical scores (CRIB and BPD classification), and standard predictors (maternal atopy, sex, PMA at birth, mechanical ventilation and weight gain). We then included lung function parameters in each of the models and compared the area under the receiver operator characteristic curve (AUC) to assess discrimination and the Brier score to assess the overall predictive performance of the models. The Brier score [23] is the averaged squared difference between the predicted and observed outcomes: the lower the score, the more accurate the prediction, so that a perfect predictor would have a score of 0 , a perfect mispredictor a score of 1 and a predictor acting as a fence-sitter a score of 0.25 . In addition, we calculated the p-values of likelihood ratio tests (LRTs) comparing models with and without lung function data included.

We performed the analysis using Stata version 11.2 for Windows (Stata Corporation, College Station, TX, USA).

\section{Results}

\section{Study population}

We performed lung function measurements in 236 preterm born infants between 1999 and 2010. 166 (70\%) caregivers returned the follow-up questionnaires (online supplementary fig. S1). In the analysis, we used data of 163 subjects with valid tidal measurement and 142 subjects with valid MBW according to ERS/ATS quality criteria [24] (tables 1 and 2, and online supplementary tables S1 and S2).

The 70 caregivers who did not complete questionnaires had infants with a more severe perinatal course: they had lower PMA at birth (mean 27.9 versus 29.2 weeks), lower Apgar score at 5 min (mean 7 versus 7.6), longer mechanical ventilation (mean 39 versus 25 days) and more severe BPD. There were no significant differences in weight (in SDS), length, CRIB score, delivery mode and most lung function parameters (table S3). The cohort differed from the total population of preterms hospitalised at the Children's Hospital of Bern in that the clinical conditions of the preterms without BPD are somewhat poorer (table S4).

\section{Pathophysiological association}

Infant lung function was weakly associated with subsequent wheeze and inhalation therapy (table 3). For example, VT was positively associated with wheeze (adjusted OR (aOR) 1.40 (95\% CI 1.04-1.90) per $5 \mathrm{~mL}$ 


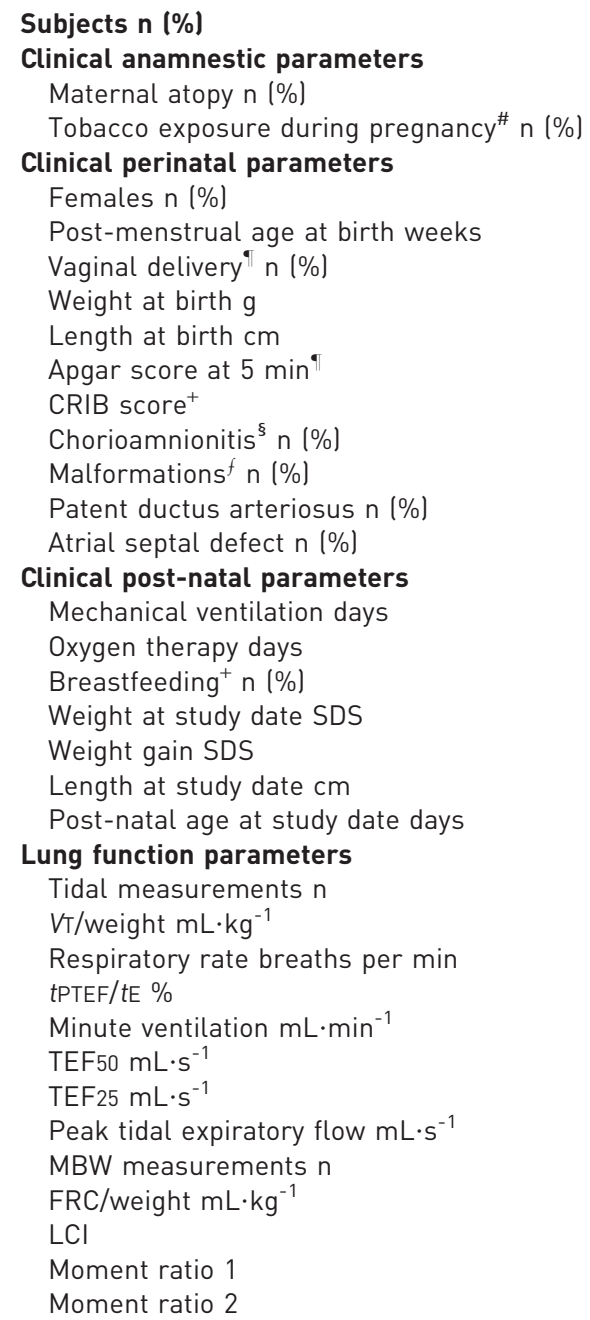

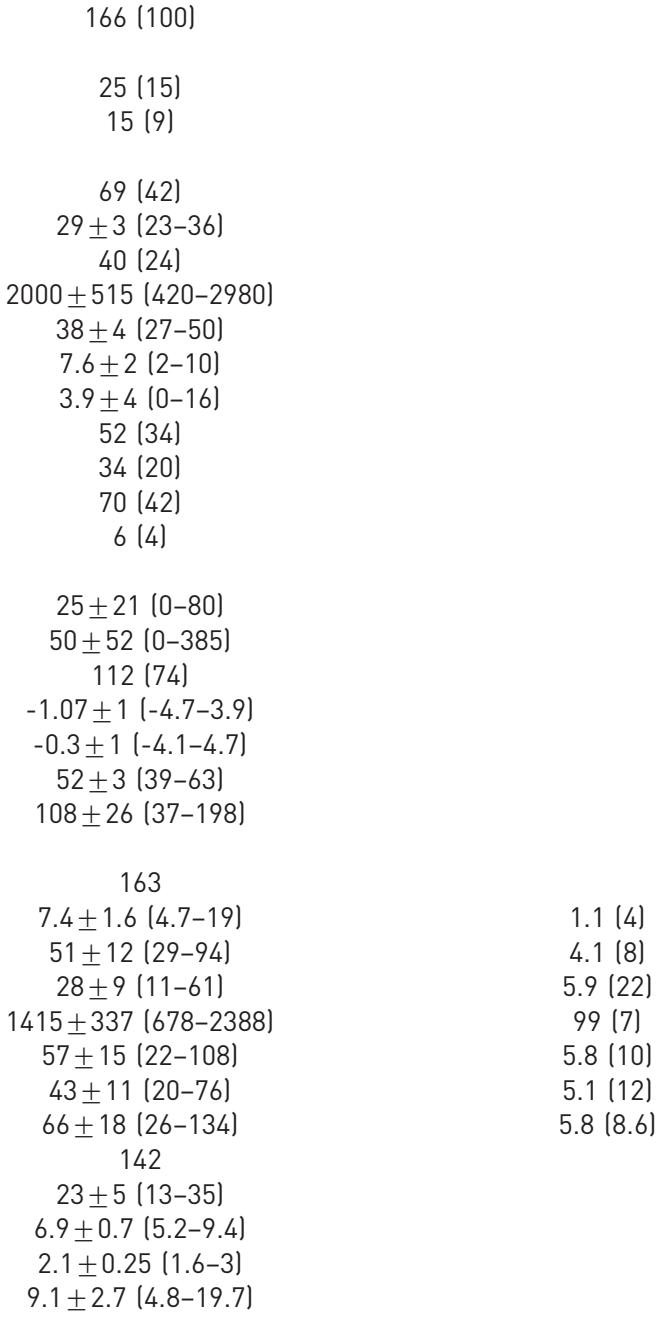

Data are presented as mean \pm SD (range) unless otherwise stated. Descriptive statistics of clinical and anamnestic data assessed in the neonatal period and lung function parameters assessed at 44 weeks of post-menstrual age. CV: coefficient of variation; CRIB: clinical risk index for babies; SDS: standard deviation score; VT: tidal volume; tPTEF: time to peak tidal expiratory flow; tE: expiratory time; TEF50: tidal expiratory flow at $50 \%$ of the remaining $V_{T}$; TEF25: tidal expiratory flow at $25 \%$ of the remaining $V_{T}$; MBW: multiple-breath washout; FRC: functional residual capacity at airway opening; LCl: lung clearance index. ${ }^{\#}$ : data missing for two subjects; ${ }^{\natural}$ : data missing for one subject; ${ }^{+}$: data missing for 15 subjects; ${ }^{\S}$ : data missing for 17 subjects; ${ }^{f}$ : including dysmorphic syndrome ( $n=3$ including subtelomere $18 q$ deletion and trisomy 21), mega cisterna magna ( $\left.n=1\right)$, plagiocephaly $(n=2)$, partial craniosynostosis $(n=1)$, macrocephaly $(n=1)$, pre-auricular tag and fistula $(n=2)$, cleft palate $(n=2)$, subglottic stenosis $(n=1)$, laryngomalacia $(n=2)$, lung hypoplasia $(n=1)$, ventricular septal defect $(n=3)$, accessory left ventricular chordae tendineae $(n=1)$, WolfParkinson-White syndrome $(n=1)$, kidney anomalies $(n=2)$, vesicoureteral reflux $(n=2)$, hydrocoele $(n=2)$, cryptorchidism ( $n=2)$, hypospadias $(n=3)$, hernia inguinalis $(n=2)$ and talipes varus $(n=1)$.

increase in $V \mathrm{~T}$ ) and respiratory rate was negatively associated with wheeze (aOR 0.69 (95\% CI 0.50-0.96) per 10 breath per min increase) (figs 1 and 2). A higher $t \mathrm{PTEF} / t \mathrm{E}$ ratio was negatively associated with several outcomes, most strongly with inhalation therapy (aOR 0.56 (95\% CI $0.35-0.89$ ) per 10\% increase) and oxygen therapy (aOR 0.27 (95\% CI 0.07-1.07) per 10\% increase). $V^{\prime}$ E, FRC, LCI, moment ratios and tidal flows (TEF50, TEF25 and peak flow) were not associated with subsequent symptoms (see table 3 for results for all lung function variables except flows).

All approaches of the sensitivity analyses confirmed the main results (online supplementary table S5). For instance, the aOR per $10 \%$ increase in $t \mathrm{PTEF} / \mathrm{tE}$ for inhalation therapy was 0.65 (95\% CI $0.43-0.97$ ) in the 


\section{TABLE 2 Outcomes: respiratory symptoms}

$\begin{array}{lc}\text { Subjects n } & 166 \\ \text { Wheeze } & 76(46) \\ \text { Inhalation therapy } & 63(38) \\ \text { Re-hospitalisation } & 41(25) \\ \text { Home oxygen therapy } & 11(6)\end{array}$

Data are presented as $\mathrm{n}(\%)$ unless otherwise stated. Symptoms during the first year of life assessed through questionnaire.

unadjusted model, 0.56 (95\% CI 0.35-0.89) in the main model and 0.54 (95\% CI 0.29-1.03) when further adjusted. Six infants required oxygen therapy at the time of lung function measurements; none was ventilated. The sensitivity analysis adjusted for additional oxygen showed comparable results. The stratification based on oxygen therapy (yes/no) at 36 weeks PMA (i.e. no or mild BPD versus moderate or severe $\mathrm{BPD}$ ) also confirmed the main results (data not shown).

\section{Clinical relevance}

The prediction model including common clinical scores only (BPD severity and CRIB score) had the lowest performance, with an AUC of 0.54 and a Brier score of 0.25 . The model with the combination of standard clinical predictors had a better performance, with an AUC of 0.67 and a Brier score of 0.23 . Adding lung function parameters to each of these three models did not improve the performance (table 4 and fig. 3 ). For example, adding $V \mathrm{~T}$, respiratory rate and $\mathrm{tPTEF} / \mathrm{tE}$ to a combination of standard clinical predictors (sex, PMA, mechanical ventilation, weight gain and maternal atopy), the AUC increased from 0.68 to 0.71 and the Brier score improved from 0.23 to 0.22 . The p-value of the LRT was 0.12 .

\section{Discussion}

\section{Summary}

With the present study, we demonstrate weak correlations of tidal breathing parameters with subsequent respiratory symptoms. These findings give interesting insight into adaptive mechanisms in preterm infants but do not help to predict respiratory outcomes in these children.

\section{Pathophysiological association}

From a physiological point of view, we observed an interesting breathing pattern among the infants developing subsequent wheeze. This group has a lower respiratory rate and a higher VT. We hypothesise that this might be caused by adaptive mechanisms only seen in unsedated infants. Greater dynamic dead space and airways more susceptible to collapse may be compensated by higher $V_{T}$ (lung volume recruitment), leading to lower respiratory rates, in order to maintain constant $V^{\prime} \mathrm{E}$. An increase in dynamically controlled dead space could lead to increased ventilation heterogeneity, i.e. increased LCI, as reported previously $[2,25]$. Additional data suggest an immature hypercapnic ventilatory response in the

TABLE 3 Associations between lung function at 44 weeks of post-menstrual age and subsequent symptoms during infancy

\begin{tabular}{|c|c|c|c|c|}
\hline & Wheeze & Inhalation therapy & Re-hospitalisation & Home oxygen therapy \\
\hline Respiratory rate per 10 breaths per min & $0.69(0.5-0.96)^{*}$ & $0.89(0.66-1.21)$ & $1.00(0.72-1.40)$ & $1.82(0.93-3.5)$ \\
\hline Tidal volume per $5 \mathrm{~mL}$ & $1.40(1.04-1.9)^{*}$ & $1.2(0.89-1.6)$ & $0.85(0.58-1.26)$ & $0.30(0.08-1.2)$ \\
\hline tPTEF/tE per $10 \%$ & $0.96(0.64-1.43)$ & $0.56(0.35-0.89) *$ & $0.72(0.43-1.18)$ & $0.27(0.07-1.07)$ \\
\hline FRC per $5 \mathrm{~mL}$ & $0.93(0.83-1.05)$ & $0.96(0.85-1.08)$ & $0.89(0.77-1.02)$ & $0.77(0.57-1.04)$ \\
\hline $\mathrm{LCl}$ & $0.71(0.42-1.2)$ & $1.15(0.66-1.99)$ & $0.52(0.27-1.0)$ & $1.6(0.49-5.3)$ \\
\hline
\end{tabular}

Data are presented as adjusted OR (95\% Cl) for symptoms per increase in lung function parameter by the increment indicated. The units were arbitrarily chosen for better readability. The multivariable regressions were adjusted for maternal atopy, sex, mode of delivery, post-menstrual age at birth, Apgar score at $5 \mathrm{~min}$, duration of mechanical ventilation, weight gain from birth until the lung function test date as a standard deviation score (SDS), and post-menstrual age, weight as a SDS and length in centimetres on the lung function test date. tPTEF: time to peak tidal expiratory flow; tE: expiratory time; FRC: functional residual capacity at airway opening; LCl: lung clearance index. ${ }^{*}: \mathrm{p}<0.05$. 

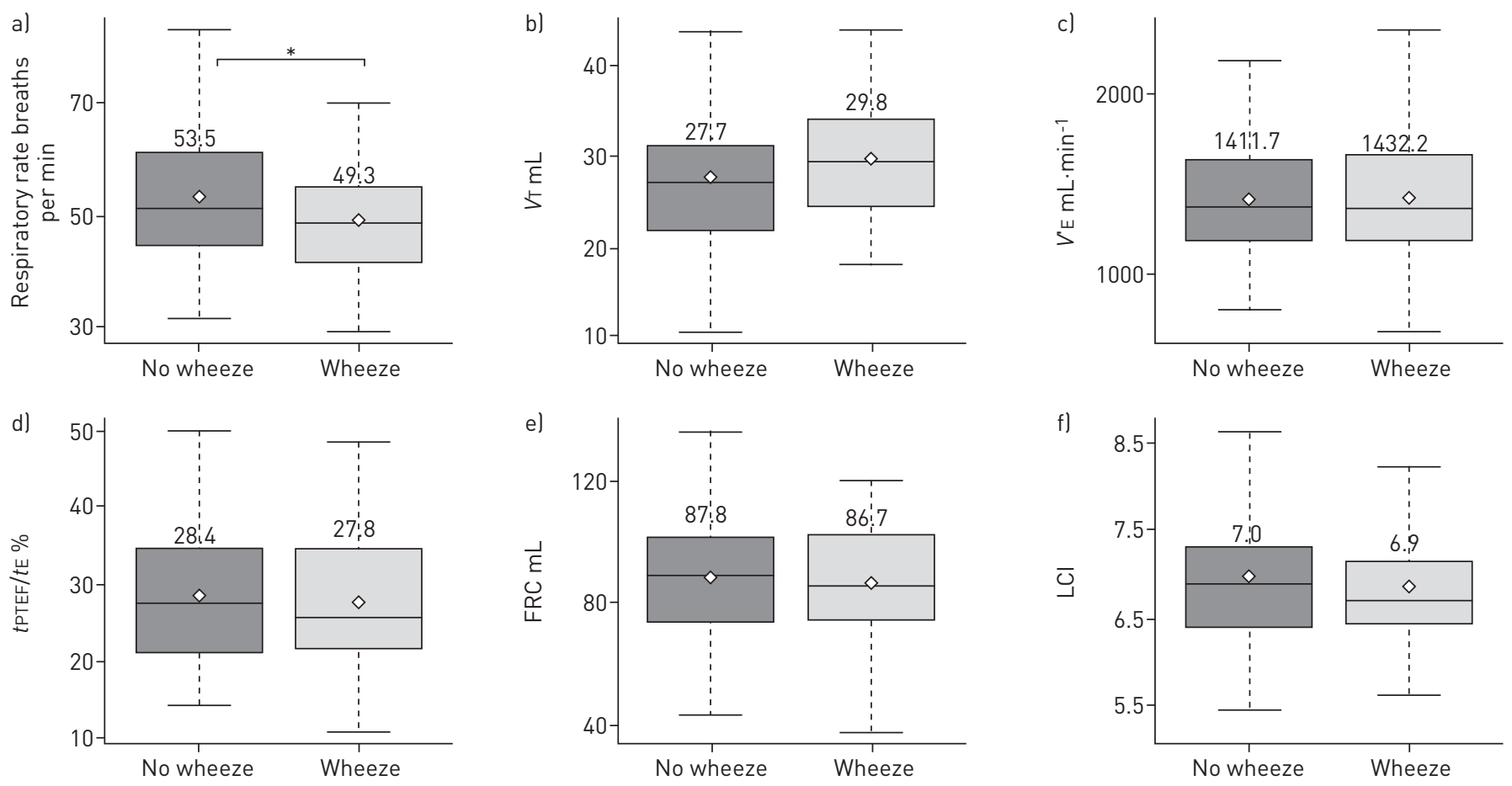

FIGURE 1 Box plots of a) respiratory rate, b) tidal volume $(V T), c)$ minute ventilation $\left(V^{\prime} \mathrm{E}\right)$, d) time to peak tidal expiratory flow $(t \mathrm{PETF}) / \mathrm{expiratory}$ time $(t \mathrm{E})$ ratio, e) functional residual capacity (FRC) and f) lung clearance index (LCI), measured at 44 weeks of post-menstrual age, stratified by the outcome wheeze. Numbers and diamonds indicate the mean value of the lung function parameter within the groups, horizontal lines represent the median, boxes represent the interquartile range, and whiskers represent the range. We performed t-tests to compare the mean values of each lung function parameter in the infants who developed subsequent wheeze and in the group who did not develop wheeze. We found a difference in respiratory rate of 4.2 breaths per min between the two groups. ${ }^{*}: \mathrm{p}<0.05$.

first weeks of life with limited change in respiratory rate but substantial increase of $V T$ [26]. Whether this immature response still influences the breathing pattern at 44 weeks PMA in premature infants remains unclear, as it has only been studied in the first few weeks of life. Another explanation for higher VT and lower respiratory rates could be a higher time constant in these infants that is already present near term. This hypothesis is supported by the work of VAN PUTTE-KATIER et al. [15], who demonstrated an increased risk of wheeze in the first year of life in term-born infants with higher time constant at the age of 2 months, and by a recent publication by DRYSDALE et al. [27], who reported that prematurely born infants admitted to hospital for lower respiratory tract infection in the first year of life had higher resistance of the respiratory system at 36 weeks PMA. To further elucidate the mechanical changes in this group of premature infants, future studies need to explore airway mechanics, flow limitation and adaptive mechanisms in more detail using additional lung function techniques.

An as yet unexplored but potentially highly relevant explanation is that preterm infants have a ventilation perfusion mismatch and a rarefication of the acinar structure. Modelling studies of pulmonary carbon dioxide washout have shown that a potential simplification of acinar structure may affect carbon dioxide clearance mostly at low $V_{\mathrm{T}}$ independently of the respiratory rate [28]. Thus, in order to maintain $V^{\prime} \mathrm{E}$ and effective carbon dioxide elimination, preterms may predominantly increase $V_{T}$ and even decrease respiratory rate.

\section{Clinical relevance}

Lung function measured at 44 weeks PMA in premature infants is weakly related to respiratory symptoms in the first year of life. The differences in $V \mathrm{~T}$, respiratory rate and $t \mathrm{PTEF} / \mathrm{tE}$ we found at the group level (between those infants who subsequently developed wheeze or needed inhalation therapy versus those who did not) had poor predictive value for later respiratory symptoms at the individual level. From a clinical point of view, the lung function parameters measured near term in our study do not yet help in predicting later respiratory symptoms in the first year of life. 


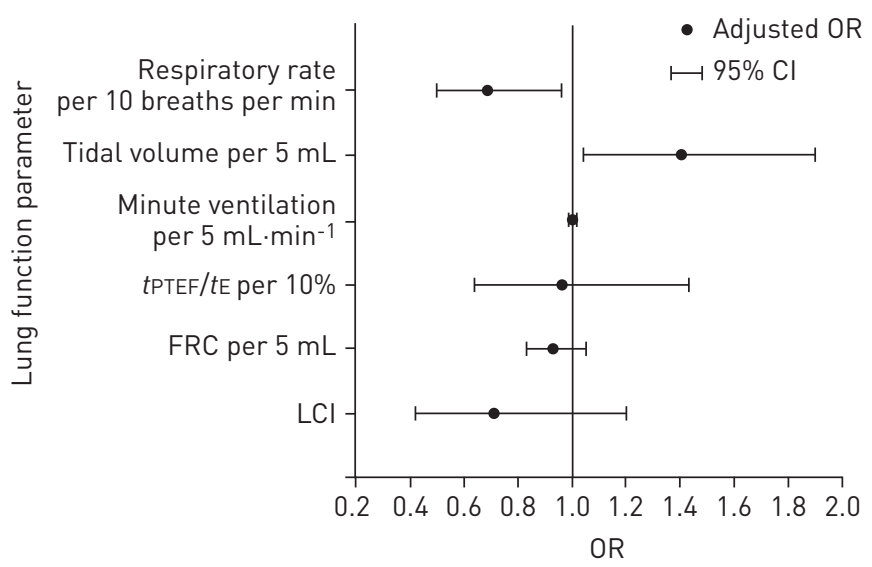

FIGURE 2 Association between the lung function parameters assessed at 44 weeks of post-menstrual age (PMA) and subsequent wheeze during infancy. The models were adjusted for maternal atopy, sex, mode of delivery, PMA at birth, Apgar score at $5 \mathrm{~min}$, duration of mechanical ventilation, weight gain as a standard deviation score (SDS) from birth until the lung function test date, and PMA, weight as SDS and length in centimetres at the lung function test date. Adjusted odds ratio and 95\% confidence intervals for developing subsequent wheeze are shown, per increment of the lung function parameter. $\mathrm{tPTEF}$ : time to peak tidal expiratory flow; tE: expiratory time; FRC: functional residual capacity; LCI: lung clearance index.

\section{Predicting respiratory outcome in preterms: comparison with previous literature}

Multiple studies aimed to predict respiratory outcome after preterm birth using different clinical factors, such as chorioamnionitis or BPD. Even prematurity without BPD is associated with a higher prevalence of respiratory symptoms in preschool children and schoolchildren [3, 4, 9, 29, 30]. Additionally, preterms can exhibit impaired lung function from preschool age to adulthood $[1,5]$. In contrast to our study, these studies used lung function as an outcome but not as a predictor.

Other studies included term-born infants and assessed whether infant lung function predicts short-term outcomes [13, 15, 31]. Greenough et al. [13] investigated whether lung function testing in term-born infants shortly after birth was predictive of respiratory outcome in the first year of life. They found that higher airway resistance and higher FRC after birth related to symptoms in the first year of life, but the positive predictive value was weak. DRYSDALE et al. [27] studied whether preterm infants developing viral lower respiratory tract infection in the first year of life had poorer lung function at 36 weeks PMA than infants without infection. They found no difference in respiratory system resistance, compliance or FRC,

TABLE 4 Additional value of adding data from lung function tests, compared to a model including standard clinical predictors only, for the prediction of wheeze during the first year of life

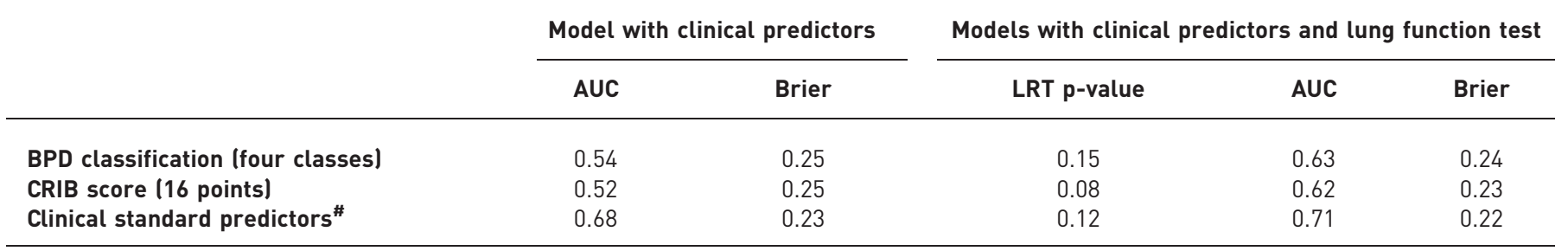

Prediction of wheeze using bronchopulmonary dysplasia (BPD) classification, clinical risk index for babies (CRIB) score, clinical standard predictors alone and with the lung function parameters associated with symptoms in the first analysis (minute ventilation, respiratory rate and time to peak tidal expiratory flow/expiratory time ratio). Area under the receiver operating characteristic curve (AUC) and Brier score of the logistic regression models including clinical parameters and with addition of lung function parameters combined are shown. The models with lung function parameters were adjusted for age and weight as a standard deviation score at the time of the lung function test. We compared the models using the likelihood ratio test (LRT). The LRT is used to compare the fits of two similar (nested) models. A p-value $<0.05$ suggests that one model fits the data significantly better than the other. The Brier score measures the total difference between the event (wheeze) and the prediction probability of that event as an average squared difference. As a benchmark, a perfect predictor would have a Brier score of 0 , a perfect mispredictor would have a Brier score of 1 and a fence-sitter (prediction of every wheezer as a "50/50" chance) would have a Brier score of 0.25 . \#: sex, post-menstrual age, maternal atopy, weight gain from birth until the time of lung function test and days of mechanical ventilation. 


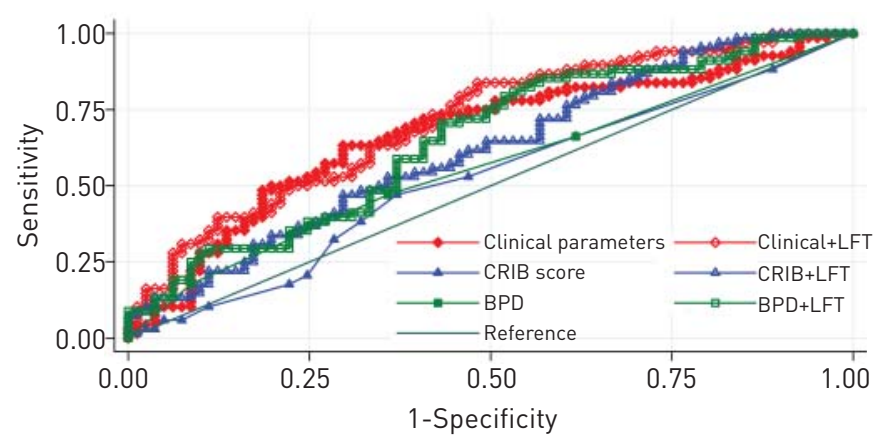

FIGURE 3 Receiver operating characteristic curves comparing bronchopulmonary dysplasia (BPD) classification, clinical risk index for babies (CRIB) score, clinical standard predictors alone and with the lung function parameters (minute ventilation, respiratory rate, time to peak tidal expiratory flow/expiratory time ratio) for prediction of subsequent wheeze during the first year of life. Clinical predictors: sex, post-menstrual age, maternal atopy, weight gain from birth until the time of lung function test and days of mechanical ventilation. LFT: lung function test.

except in children who were hospitalised, whose resistance was significantly higher. It is important to note that measurements of lung function at 36 weeks PMA are technically demanding, show high variability and represent different aspects of lung mechanics to measurements performed in later phases of lung development after term. In contrast to our study, DRYSDALE et al. [27] used a combined outcome of cough, wheeze and/or shortness of breath, and they used different lung function techniques, which makes comparison with our data difficult. Consistent with our data, VAN PUTTE-KATIER et al. [15] showed in termborn infants that a higher respiratory system compliance before the age of 2 months was associated with a decreased risk of cough and wheeze during the first year of life, whereas higher resistance and time constant were associated with an increased risk of cough and wheeze. KAVVADIA et al. [31] investigated the predictive value of very early (day 2 of life) lung function measurements and found that FRC $<19 \mathrm{~mL} \cdot \mathrm{kg}^{-1}$ body weight was independently predictive of oxygen dependency beyond day 28 or 36 weeks PMA in very preterm infants. In summary, there is little evidence to support regular clinical use of current lung function techniques to estimate respiratory disease outcomes in premature infants.

\section{Strengths and limitations}

We investigated the clinical value of early lung function measurements to predict respiratory morbidity during the first year of life in preterm infants. To our knowledge, together with the study by DRYSDALE et al. [27], this is the only large study in preterm infants. Ideal for prediction would be a lung function parameter with low variability, such as maximal flow at FRC; however, these techniques are more invasive as they require sedation for squeeze manoeuvres and are less applicable in sick infants, as a bedside application or for widespread clinical use. We used a noninvasive technique not interfering with potential physiological adaptations of underlying structural and/or functional alterations, being aware of the potentially larger intersubject variability. At 44 weeks PMA, high-quality, standardised lung function measurements are easier to perform, compared with measurements at 36 weeks PMA [18]. Nevertheless, normal physiological variability is still rather high, making infant lung function in an individual difficult to interpret.

Retrospective questionnaires always carry the risk of reporting bias. The dropout rate of $30 \%$ in the first year of life is comparable to that reported in other studies [3]. However, it entails the risk of selection bias. In fact, the final cohort contained fewer subjects with severe BPD. As the aim of this study was to investigate an association between lung function and symptoms, and not to obtain precise estimates of prevalence or incidence of symptoms, this should not have greatly affected our findings. This is further supported by the fact that results in the subgroup of children with more severe BPD were comparable to those from the whole group. Moreover, the preterms with BPD had a comparable clinical condition to those in the whole population of preterms. We did not include more specific lung function measures that would require tidal phase III slope analyses (e.g. double-tracer gas phase III slope, and ventilation inhomogeneity of conducting and acinar airways).

\section{Conclusion}

Alterations in tidal breathing pattern measured at 44 weeks were associated with respiratory outcomes in the first year of life in premature infants. For instance, higher $V \mathrm{~T}$ and lower respiratory rate were associated with wheeze later in life. This combination potentially reflects an adaptive change in breathing pattern due to underlying structural pathology. However, the clinical value of postnatal lung function testing for the prediction of respiratory morbidity is limited for the individual child: lung function data (tidal breathing 
and MBW measurement) did not improve prediction of morbidity over and above standard clinical parameters, which are easier to assess. This could partly be due to the high variability of parameters measured during natural sleep. Whether this is different for more invasive lung function tests, such as raised volume rapid thoracic compression, needs to be examined; however, their invasive nature and the need for sedation preclude clinical application in routine settings.

In summary, we do not recommend routine tidal lung function testing as a clinical tool to predict subsequent symptoms in the first year of life, but we strongly recommend its use in future studies as an important means for understanding respiratory pathophysiology.

\section{Acknowledgements}

We thank Olaf Ahrens and Roland Gerull (Division of Neonatology, Department of Paediatrics, University Children's Hospital Bern, Bern, Switzerland) who provided us with neonatal data; Gisela Wirz, Monika Graf, Sandra Lüscher and Barbara Hofer (study nurses; UKBB, Basel, Switzerland), who were responsible for data management and who were largely responsible for performing lung function measurements; and Janet Maccora (UKBB), who provided Englishlanguage editorial assistance.

\section{References}

1 Baraldi E, Filippone M, Trevisanuto D, et al. Pulmonary function until two years of life in infants with bronchopulmonary dysplasia. Am J Respir Crit Care Med 1997; 155: 149-155.

2 Latzin $\mathrm{P}$, Roth S, Thamrin C, et al. Lung volume, breathing pattern and ventilation inhomogeneity in preterm and term infants. PLoS One 2009; 4: e4635.

3 Vrijlandt EJ, Kerstiens JM, Duiverman EJ, et al. Moderately preterm children have more respiratory problems during their first 5 years of life than children born full term. Am J Respir Crit Care Med 2013; 187: 1234-1240.

4 Narang I, Rosenthal M, Cremonesini D, et al. Longitudinal evaluation of airway function 21 years after preterm birth. Am J Respir Crit Care Med 2008; 178: 74-80.

5 Doyle LW, Faber B, Callanan C, et al. Bronchopulmonary dysplasia in very low birth weight subjects and lung function in late adolescence. Pediatrics 2006; 118: 108-113.

6 Schmalisch G, Wilitzki S, Wauer RR. Differences in tidal breathing between infants with chronic lung diseases and healthy controls. BMC Pediatr 2005; 5: 36.

7 Pramana IA, Latzin P, Schlapbach LJ, et al. Respiratory symptoms in preterm infants: burden of disease in the first year of life. Eur I Med Res 2011; 16: 223-230.

8 Ehrenkranz RA, Walsh MC, Vohr BR, et al. Validation of the National Institutes of Health consensus definition of bronchopulmonary dysplasia. Pediatrics 2005; 116: 1353-1360.

9 Lamarche-Vadel A, Blondel B, Truffer P, et al. Re-hospitalization in infants younger than 29 weeks' gestation in the EPIPAGE cohort. Acta Paediatr 2004; 93: 1340-1345.

10 Vrijlandt EJLE, Duiverman EJ. Pulmonary function testing in premature infants and infants with bronchopulmonary dysplasia, In: Frey U, Merkus PJFM, eds. Paediatric Lung Function. Eur Respir Monogr 2010; 47: 251-262.

1 Gabriele C, Jaddoe VW, van Mastrigt E, et al. Exhaled nitric oxide and the risk of wheezing in infancy: the Generation R Study. Eur Respir J 2012; 39: 567-572.

12 Giffin F, Greenough A, Yuksel B. Relationship between lung function results in the first year of life and respiratory morbidity in early childhood in patients born prematurely. Pediatr Pulmonol 1994; 18: 290-294.

13 Greenough A, Naik S, Itakura Y, et al. Perinatal lung function measurements and prediction of respiratory problems in infancy. Physiol Meas 1998; 19: 421-426.

14 Latzin P, Kuehni CE, Baldwin DN, et al. Elevated exhaled nitric oxide in newborns of atopic mothers precedes respiratory symptoms. Am J Respir Crit Care Med 2006; 174: 1292-1298.

15 van Putte-Katier N, van der Gugten AC, Uiterwaal CS, et al. Early life lung function and respiratory outcome in the first year of life. Eur Respir J 2012; 40: 198-205.

16 Frey U, Stocks J, Coates A, et al. Specifications for equipment used for infant pulmonary function testing. ERS/ATS Task Force on Standards for Infant Respiratory Function Testing. European Respiratory Society/American Thoracic Society. Eur Respir J 2000; 16: 731-740.

17 Fuchs O, Latzin P, Kuehni CE, et al. Cohort profile: the Bern infant lung development cohort. Int J Epidemiol 2012; 41: 366-376.

18 Fuchs O, Latzin P, Thamrin C, et al. Normative data for lung function and exhaled nitric oxide in unsedated healthy infants. Eur Respir J 2011; 37: 1208-1216.

19 The International Neonatal Network. The CRIB (clinical risk index for babies) score: a tool for assessing initial neonatal risk and comparing performance of neonatal intensive care units. Lancet 1993; 342: 193-198.

20 Peacock JL, Lo JW, D'Costa W, et al. Respiratory morbidity at follow-up of small-for-gestational-age infants born very prematurely. Pediatr Res 2013; 73: 457-463.

21 Strippoli MP, Silverman M, Michel G, et al. A parent-completed respiratory questionnaire for 1-year-old children: repeatability. Arch Dis Child 2007; 92: 861-865.

22 Latzin P, Frey U, Roiha HL, et al. Prospectively assessed incidence, severity, and determinants of respiratory symptoms in the first year of life. Pediatr Pulmonol 2007; 42: 41-50.

23 Pescatore AM, Dogaru CM, Duembgen L, et al. A simple asthma prediction tool for preschool children with wheeze or cough. J Allergy Child Immunol 2014; 133: 111-118.

24 Robinson PD, Latzin P, Verbanck S, et al. Consensus statement for inert gas washout measurement using multipleand single- breath tests. Eur Respir J 2013; 41: 507-522.

25 Hulskamp G, Lum S, Stocks J, et al. Association of prematurity, lung disease and body size with lung volume and ventilation inhomogeneity in unsedated neonates: a multicentre study. Thorax 2009; 64: 240-245.

26 Putnam RW, Conrad SC, Gdovin MJ, et al. Neonatal maturation of the hypercapnic ventilatory response and central neural $\mathrm{CO}_{2}$ chemosensitivity. Respir Physiol Neurobiol 2005; 149: 165-179. 
Drysdale SB, Wilson T, Alcazar M, et al. Lung function prior to viral lower respiratory tract infections in prematurely born infants. Thorax 2011; 66: 468-473.

28 Neufeld GR, Schwardt JD, Gobran SR, et al. Modelling steady state pulmonary elimination of $\mathrm{He}, \mathrm{SF}_{6}$ and $\mathrm{CO}_{2}$ : effect of morphometry. Respir Physiol 1992; 88: 257-275.

29 Palta M, Sadek-Badawi M, Sheehy M, et al. Respiratory symptoms at age 8 years in a cohort of very low birth weight children. Am J Epidemiol 2001; 154: 521-529.

30 Vrijlandt EJ, Boezen HM, Gerritsen J, et al. Respiratory health in prematurely born preschool children with and without bronchopulmonary dysplasia. J Pediatr 2007; 150: 256-261.

31 Kavvadia V, Greenough A, Dimitriou G. Early prediction of chronic oxygen dependency by lung function test results. Pediatr Pulmonol 2000; 29: 19-26. 